\title{
METODOLOGIA HEURÍSTICA CONSTRUTIVA PARA ALOCAÇÃO DE BANCOS DE CAPACITORES EM SISTEMAS DE DISTRIBUIÇÃO DE ENERGIA ELÉTRICA
}

\author{
Ivo Chaves da Silva Junior* \\ ivojeufrj.br \\ Edimar José de Oliveira ${ }^{\dagger}$ \\ edimarlacee.ufjf.br
}

\author{
Sandoval Carneiro Junior* \\ sandoval@dee.ufrj.br
}

José Luiz Rezende Pereira ${ }^{\dagger}$

jluiz@ieee.org

\section{Paulo Augusto Nepomuceno Garcia ${ }^{\dagger}$ \\ pgarcia@lacee.ufjf.br}

*Universidade Federal do Rio de Janeiro-RJ

${ }^{\dagger}$ Universidade Federal de Juiz de Fora-MG

\section{RESUMO}

Este artigo apresenta um algoritmo heurístico construtivo para a resolução do problema referente à alocação ótima de bancos fixos de capacitores em sistemas de distribuição. Inicialmente a natureza discreta do problema de alocação de capacitores é mitigada através do uso de uma função sigmoidal no intervalo [0,1] que é incorporada ao problema de fluxo de potência ótimo (FPO) através de modificações nas equações de fluxo de potência. $\mathrm{O}$ algoritmo proposto utiliza a técnica primal-dual de pontos interiores como ferramenta de solução do FPO. Baseado na sensibilidade fornecida pela função sigmoidal ponderada pelo carregamento da potência reativa do sistema de distribuição, a metodologia heurística proposta determina passo a passo a localização, a capacidade e o número de capacitores a serem instalados no sistema. A técnica proposta é aplicada a dois sistemas de distribuição de energia. Os resultados são comparados com os resultados obitidos através de um algoritmo genético.

PALAVRAS-CHAVE: Alocação Ótima de Capacitores, Algo-

\footnotetext{
Artigo submetido em 10/02/2006

1a. Revisão em 26/10/2006

2a. Revisão em 15/10/2007

Aceito sob recomendação do Editor Associado

Prof. Denis Vinicius Coury
}

ritmo Heurístico Construtivo, Função Sigmoidal, Técnica de Pontos Interiores.

\section{ABSTRACT}

This paper presents a heuristic constructive algorithm to solve the discrete capacitor size and allocation problem in distribution systems. Initially the discrete nature of the capacitor allocation problem is mitigated by using a sigmoid function in the $[0,1]$ interval which is incorporated into the optimum power flow (OPF) through modifications in the power flow equations. The proposed algorithm uses the primal-dual interior point technique to solve the OPF problem. Finally, the sensitivity obtained by sigmoid function which is weighted by the reactive loading of the distribution system, determines step by step the location, size and number of capacitors to be installed in the distribution system. The proposed technique is applied in two distribution systems. The results obtained are compared with the ones obtained using the genetic algorithm.

KEYWORDS: Optimal Capacitor Allocation, Heuristic Constructive Algorithm, Sigmoid Function, Interior Point Technique. 


\section{INTRODUÇÃO}

Com o novo modelo do setor elétrico, as concessionárias de energia passaram a investigar a operação otimizada de seus sistemas de distribuição, principalmente no que diz respeito à redução das perdas de energia nos alimentadores. Tendo em vista que estes sistemas apresentam um valor elevado de perdas de energia e que a redução destas perdas possui impacto no valor das tarifas, vários estudos relativos à minimização de perdas estão recebendo atenção especial por parte de engenheiros e técnicos do setor. Uma das soluções mais utilizadas para a redução das perdas de energia é a alocação de capacitores no sistema de distribuição, ou seja, determinação dos locais, tamanhos, tipos e números de capacitores a serem instalados no sistema. A alocação de bancos de capacitores proporciona grandes benefícios não só ao problema referente às perdas de energia como também melhora o perfil de tensão e o fator de potência, liberando a capacidade do sistema (Grainger e Lee, 1981; Lee e Grainger, 1981; Grainger e Lee, 1982)

O problema de alocação de capacitores se caracteriza como um problema de otimização não linear inteira mista de difícil solução e que apresenta dois fatores que adicionam mais complexidade ao problema: (i) Existência de várias soluções factíveis, ou seja, tem-se uma região de solução não convexa apresentando vários mínimos locais, dificultando a obtenção da solução mínima global; (ii) Elevado tempo computacional, ou seja, tem-se uma explosão combinatória referente às alternativas discretas de investimento.

Na literatura são encontrados vários algoritmos desenvolvidos para a resolução do problema de alocação ótima de capacitores. Nos últimos anos a aplicação de algoritmos genéticos vem sendo bastante utilizada (Salama e Chikhani, 2000; Delfanti et al, 2000; Santos et al, 2004) principalmente pelo fácil tratamento das variáveis discretas e pela qualidade das soluções obtidas. Entretanto, esta técnica tem como desvantagem o elevado tempo de processamento (Levitin et al, 2000). Por outro lado, os algoritmos heurísticos construtivos (Garver, 1970; Monticelli et al, 1982; Villasana et al, 1985), são robustos, de fácil entendimento e apresentam baixo esforço computacional, porém a principal desvantagem é a qualidade das soluções obtidas, principalmente para sistemas de grande porte. Ou seja, o ponto ótimo global dificilmente é encontrado.

No entanto, as referências (Oliveira et al, 2005; Gomes et al 2005) apresentaram heurísticas capazes de encontrar soluções de qualidade, inclusive para sistema de grande porte. Em (Oliveira et al, 2005) a natureza discreta, inerente ao problema do planejamento da expansão de sistemas de transmissão, foi abordada com sucesso através do tratamento das variáveis discretas com base na sensibilidade fornecida por uma função sigmoidal. Esta representação tem como característica principal robustez e baixo tempo de processamento.

Assim, diante dos resultados obtidos, optou-se no presente trabalho por utilizar a função sigmoidal para modelar a inserção ou não dos bancos de capacitores no sistema de distribuição de energia.

Na metodologia proposta, a função sigmoidal é incorporada ao problema do fluxo de potência ótimo através de modificações nas equações do fluxo de carga. Desta forma, o problema que originalmente é de programação não linear inteira mista, passa a ser inicialmente um problema de programação não linear contínua. O processo de decisão, referente à capacidade, localização e número de capacitores instalados, é realizado através de uma heurística baseada na sensibilidade fornecida pela função sigmoidal ponderada pelo carregamento de potência reativa do sistema de distribuição. Com isso, evita-se a explosão combinatória e o elevado tempo computacional. A técnica apresentada é inexplorada na literatura técnica especializada para alocação de bancos de capacitores, o que caracteriza um campo fértil para a pesquisa.

\section{FORMULAÇÃO PROPOSTA}

Tradicionalmente, o problema do fluxo de potência ótimo referente ao estudo da alocação ótima de capacitores é formulado como um problema de programação não linear, contendo variáveis contínuas, como tensões, ângulos de potência, potências geradas (ativas e reativas) e variáveis discretas, como a decisão de inserção ou não dos bancos de capacitores no sistema de distribuição de energia.

Esta decisão de inserir ou não determinado banco de capacitores é similar ao funcionamento de uma chave lógica, ou seja: $(i)$ chave $=0$, banco não inserido; (ii) chave $=1$, banco inserido. A figura 1 ilustra a possibilidade da inclusão de um banco de capacitores em uma barra $i$ de um sistema de distribuição de energia, onde a chave representa a variável discreta do problema, ou seja, a decisão de inserção ou não do banco de capacitores.

A modelagem para a representação do chaveamento dos bancos é dada pela função degrau unitário, uma vez que esta assume dois valores distintos (0-1) que representam o funcionamento de uma chave lógica. Entretanto sua aplicação direta no FPO é inviável pelo fato desta função ser descontínua. Diante disso, adotou-se uma função sigmoidal para modelar o chaveamento do banco de capacitores, sendo esta função diferenciável e com um comportamento similar ao da curva característica da função degrau unitária. Neste caso, esta função apresenta forte sensibilidade para a representação dos dois estados (0-1) inerentes ao chaveamento, como apresentado na figura 2 . 


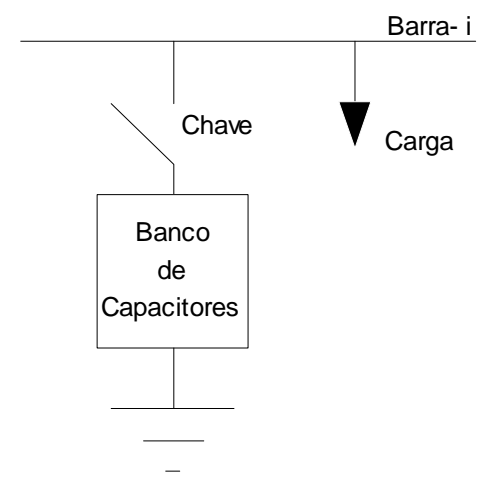

Figura 1: Chaveamento do Banco de Capacitor.

As funções sigmoidais são normalmente aplicadas em trabalhos envolvendo redes neurais como funções de ativação na modelagem de neurônios artificiais (Haykin, 1998). A função sigmoidal adotada neste trabalho é expressa pela Equação (1).

$$
\operatorname{chave}(x)=C H(x)=\frac{e^{x}-1}{e^{x}+1} \mathrm{p} / \mathrm{x} \geq 0
$$

A função sigmóide proposta $(\mathrm{CH})$ é introduzida na tradicional formulação do FPO através do produto entre o valor do banco de capacitor a ser instalado na barra- $i\left(\mathrm{Q}_{C i}\right)$ e o valor da chave de conexão do capacitor $\left(\mathrm{CH}_{i}\right)$ na barra- $i$. Ou seja:

$$
Q_{i}=Q c_{i} \cdot C H_{i}(x)
$$

onde:

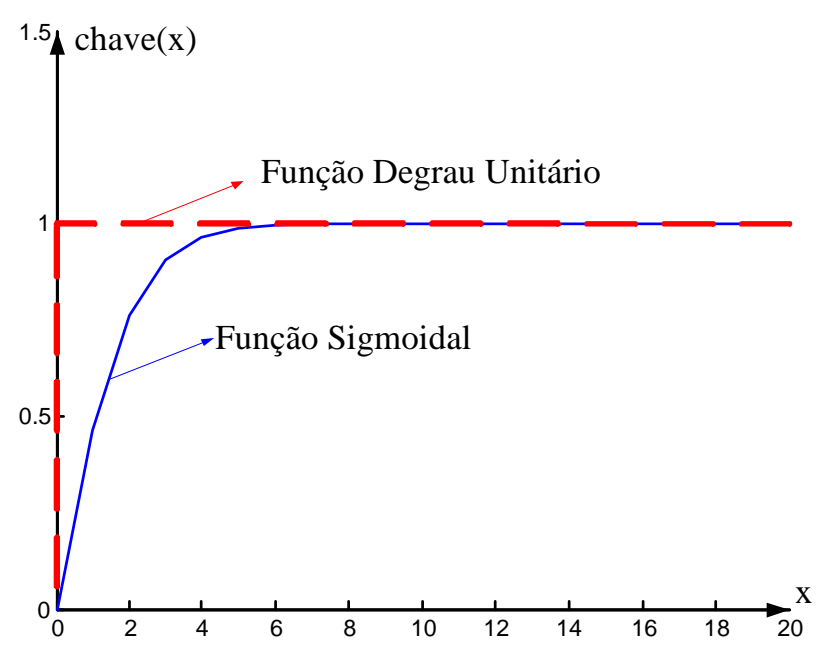

Figura 2: Modelagem do Chaveamento.
$Q_{i}$ É o valor da potência reativa injetada pela inserção do banco de capacitor localizado na barra $i$.

Introduzindo este conceito na tradicional formulação do FPO, tem-se:

$$
\operatorname{Min}\left[\sum_{k=1}^{\mathrm{nc}} \text { ce.T. } L_{k}+\sum_{i=1}^{\mathrm{nb}} \mathrm{cb} \cdot \mathrm{CH}_{\mathrm{i}}(\mathrm{x}) \cdot \mathrm{Qc} \mathrm{c}_{\mathrm{i}}\right]
$$

Sujeito a:

$$
\begin{gathered}
P g_{i}-P l_{i}+\sum_{j \in \Omega i}^{p_{i j}=0} \\
Q g_{i}+\left(C H_{i}(x) \cdot Q c_{i}\right)-Q l_{i}+\sum_{j \in \Omega i}^{q_{i j}=0} \\
L=\sum_{j \in \Omega i} g_{i j} \cdot\left[V_{i}^{2}+V_{j}^{2}-2 \cdot V_{i} \cdot V_{j} \cdot \cos \left(\theta_{i j}\right)\right] \\
p_{i j}^{\min } \leq p_{i j} \leq p_{i j}^{\max } \\
\frac{0}{\frac{Z^{\min }}{} \leq \bar{Z} \leq \overline{Z^{\max }}}
\end{gathered}
$$

onde:

nc Número de circuitos;

ce Custo da energia (US\$/kWh);

$T$ Período de carregamento (horas);

$L_{k}$ Perda de potência ativa para o circuito $k$;

$c b$ Custo do banco de capacitor (US $\$ / \mathrm{kVAr}$ );

$C H_{i}(x)$ Função sigmoidal, representando a inserção $(\mathrm{CH}=$ 1) ou não $(\mathrm{CH}=0)$ do banco de capacitor, na barra $i$;

$x$ Argumento da função sigmoidal;

$Q c_{i}$ Valor do banco de capacitores a ser instalado ou não na barra $i$;

$P g_{i}$ Potência ativa gerada na barra $i$;

$P l_{i}$ Carga ativa na barra $i$;

$p_{i j}$ Fluxo de potência ativa entre as barras $i-j$;

$Q g_{i}$ Potência reativa gerada na barra $i$;

$Q l_{i}$ Carga reativa na barra $i$

$q_{i j}$ Fluxo de potência reativa entre as barras $i-j$;

$V_{i}^{\prime} V_{j}$ Módulo de tensão nas barras $i$ e $j$ respectivamente; 
$g_{i j}$ Condutância do circuito localizado entre as barras $i-j$;

$\theta_{i j}$ Diferença angular entre as barras $i-j$.

$\bar{Z}$ Vetor correspondente as variáveis de otimização com limites de canalização;

O primeiro termo da função objetivo (3) corresponde a minimização do custo das perdas ativas, o segundo termo representa o custo do banco de capacitores. Observa-se que $\mathrm{CH}_{i}(x)=0$, nenhum investimento em fonte de potência reativa será realizado na barra i. As equações (3.1) e (3.2) correspondem às equações de balanço de potência ativa e reativa, respectivamente. Sendo a equação (3.2) modificada pela inclusão da modelagem do chaveamento dos bancos de capacitores. A equação (3.3) é referente a perda de potência ativa no circuito entre as barras $i-j$ do sistema de distribuição de energia. A restrição funcional (3.4) corresponde ao limite de fluxo de potência ativa no circuito entre as barras $i-j$ do sistema de distribuição de energia. A restrição funcional (3.5) corresponde ao limite adotado para o argumento da função sigmoidal. As restrições (3.6) representam os limites de canalização das variáveis de otimização, tais como: limites de geração ativa e reativa, de tensão e etc.

Diante da modelagem utilizada para o chaveamento, a expressão (3) representa um problema de otimização não linear contínuo, que é resolvido utilizando-se o método de pontos interiores(Karmarkar, 1991; Granville et al, 1996). Após a resolução do problema de FPO, verifica-se que a variável que representa o chaveamento do banco de capacitor pode assumir qualquer valor entre zero e um. Tendo em vista que estes valores não são realizáveis, torna-se necessário um passo adicional para decisão de quais chaves devem assumir valores unitários e quais devem assumir valores nulos. Para tanto, este trabalho propõe um algorítmo heurístico construtivo baseado nas sensibilidades das variáves proporcionadas pela solução do FPO (3).

\section{ALGORITMO HEURÍSTICO PROPOSTO}

O algoritmo proposto é resolvido em três etapas: $(i)$ etapa contínua; (ii) etapa heurística e (iii) etapa discreta, conforme o fluxograma apresentado na figura 3. A alocação realizada neste trabalho adota os bancos de capacitores fixos.

Inicialmente, um FPO é executado considerando o sistema de distribuição original, isto é, sem a inclusão de nenhuma fonte de potência reativa. Isso é feito com o objetivo de verificar as perdas iniciais do sistema e principalmente o valor da função objetivo referente à minimização do custo das perdas, primeiro termo da Equação (3). Isto se faz necessário porque este valor é utilizado no primeiro passo do algoritmo como critério de decisão do processo de alocação. Com o

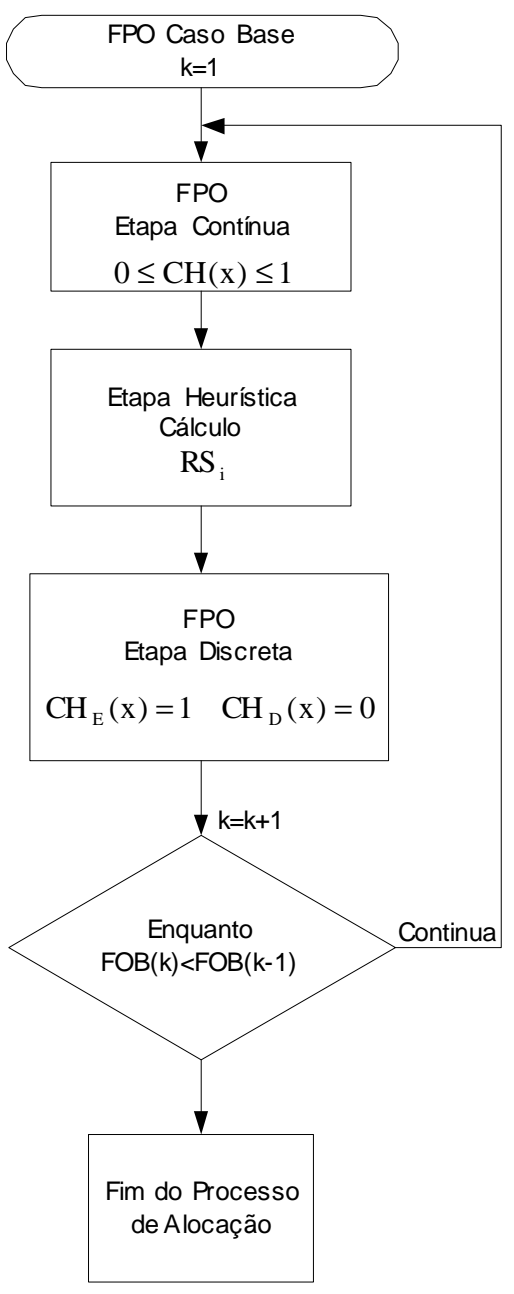

Figura 3: Fluxograma do Algoritmo Proposto

conhecimento do custo total das perdas de potência ativa nos alimentadores, inicia-se a primeira etapa do algoritmo proposto, ou seja, a etapa contínua.

\subsection{Etapa Contínua}

A etapa contínua consiste na execução do FPO (3) permitindo que $C H(x)$ assuma valores contínuos dentro do intervalo $0 \leq C H(x) \leq 1$. Para tanto, tem-se o argumento (x) da função sigmoidal variando entre $0 \leq x \leq 20$. Esta etapa tem como objetivo a obtenção dos valores contínuos das chaves, uma vez que estes valores são utilizados na etapa subseqüente, etapa heurística, para verificar a sensibilidade da localização do banco de capacitores. 


\subsection{Etapa Heurística}

A etapa heurística tem como objetivo decidir quais são os bancos de capacitores que devem ser inseridos no sistema de distribuição. Esta decisão é realizada através da sensibilidade fornecida pela função sigmoidal. Adicionalmente, considera-se na formação da sensibilidade o valor da carga reativa da barra. Isto é importante porque a corrente e a potência aparente são dependentes da carga reativa da barra. A Equação (4) mostra a Relação de Sensibilidade (RS) adotada:

$$
\mathrm{RS}_{i}=\mathrm{Ql}_{i} \cdot \mathrm{CH}_{i}
$$

O banco de capacitores que apresentar o maior valor dado pela heurística de decisão (RS) será inserido definitivamente no sistema de distribuição.

\subsection{Etapa Discreta}

Na etapa discreta, impõe-se valor unitário à chave que conecta a rede de distribuição ao banco de capacitores que foi escolhido na etapa heurística, $C H_{E}(x)=1$. Adicionalmente, impõe-se valores nulos para as demais chaves $C H_{D}(x)=0$. A partir destas considerações o FPO (3) é novamente executado e servirá como condição inicial para a próxima etapa contínua.

É importante mencionar que as discretizações das chaves são realizadas através da alteração dos limites do argumento (x) da função sigmoidal. Assim, tem-se o argumento da função sigmoidal variando no intervalo entre $11 \leq x \leq 20$ para os bancos selecionados, ou seja, $C H_{E}(x)=1$. Para os bancos não selecionados tem-se o argumento da função sigmoidal variando entre $0 \leq x \leq 0,001$, ou seja, $C H_{D}(x)=0$. Veja figura 2.

Com estes procedimentos, o resultado do FPO mostra o novo valor da função objetivo incorporando o investimento realizado. Assim, enquanto o custo total do sistema de distribuição puder ser reduzido com a inserção dos bancos de capacitores, o processo de alocação continua. Caso contrário, o processo termina.

\section{NÍVEIS DE CARREGAMENTO}

Na literatura especializada (Huang et al, 1996), geralmente, o processo de alocação de capacitores é realizado para três níveis de carregamento: (i) Carga leve; (ii) Carga média e (iii) Carga pesada. Assim, deve-se encontrar uma proposta única de solução que contemple os três níveis de carregamento tendo em vista que neste artigo se considera o banco de capacitores fixos. Entretanto, como o enfoque principal deste trabalho está relacionado com o tratamento do pro- blema discreto de alocação, adotou-se como solução global do problema a solução encontrada para a carga média porque está carga é a predominante durante o dia.

\section{ESPAÇO DE BUSCA}

Outro aspecto importante do problema de alocação de capacitores é a questão relacionada com a dimensão da região de solução (Gallego et al, 2001; Huang et al, 1996), uma vez que esta aumenta, em grande parte dos algoritmos, a dificuldade de convergência e o tempo computacional. Diante disso, a metodologia proposta utiliza três importantes informações, obtidas da solução do FPO no caso base, a fim de construir um índice de sensibilidade adequado para reduzir o espaço de busca:

(i) Os valores dos multiplicadores de Lagrange associados às equações de balanço de potência reativa $(\lambda q)$ :] Estes multiplicadores retratam a variação da função objetivo (minimização de perdas) em relação a variação de potência reativa nas barras;

(ii) Os valores das cargas reativas (Ql):] A inserção dos bancos tem maior influência em barras de maior carga;

(iii) Os níveis de tensão $(V)$ :] Baixos valores de tensão indicam a necessidade de suporte de potência reativa, logo estes valores também são usados para compor o índice global.

Com estas informações, formulou-se a seguinte expressão:

$$
S_{i}=\frac{Q l_{i} \cdot \lambda q_{i}}{V_{i}}
$$

onde:

$\mathbf{S}_{i}$ É o índice de sensibilidade para a seleção inicial das barras. $\mathrm{O}$ índice $i$ corresponde à barra $i$.

As barras que apresentarem os maiores valores para o índice de sensibilidade $S$ serão selecionadas como candidatas à alocação dos bancos de capacitores. Entretanto, através de vários testes realizados observou-se que as barras de maior carregamento, próximas às barras indicadas pelo índice de sensibilidade $\mathrm{S}$, devem fazer parte do conjunto de candidatas à alocação dos capacitores, uma vez que estão eletricamente próximas e por isso são potencialmente candidatas. Neste trabalho adotou-se como região de busca o conjunto de barras formado por aproximadamente $20 \%$ das barras de maior sensibilidade e suas vizinhas. Este valor mostrou-se eficiente, em todos os casos estudados, para garantir que o conjunto de barras selecionado contenha a solução esperada. 


\section{ESTUDO DE CASOS}

A metodologia proposta é aplicada a dois sistemas equilibrados de distribuição de energia: $(i)$ sistema de 14 barras; (ii) sistema de 70 barras. Com base em (Baran e Wu, 1989) foram adotados os seguintes parâmetros de custos para as simulações realizadas: (i) 4 US\$/kVAr instalado; (ii) 0,06 US $\$ / \mathrm{kWh}$ referente ao custo de energia.

Com o objetivo de verificar a qualidade das soluções encontradas pela metodologia heurística proposta, os resultados são comparados com os resultados obtidos através do uso de um algoritmo genético (AG), uma vez que a aplicação desta meta-heurística é bastante difundida na literatura. Uma tarefa dificil é a determinação dos parâmetros genéticos, pois estes são de extrema relevância na qualidade das soluções obtidas pelo AG. Diante deste quadro, simulações foram realizadas e os seguintes valores para os parâmetros genéticos foram adotados: (i)taxa de cruzamento de $90 \%$; (ii) taxa de mutação de 2\%; (iii) população de 200 indivíduos; (iv) 100 gerações; $(v)$ critério de convergência baseado no número máximo de gerações; (vi) elitismo;(vii) codificação binária dos indivíduos ; (viii) geração randômica da população inical; ( $i x)$ seleção via roleta e $(x)$ cruzamento em dois pontos de corte.

Todas as simulações foram realizadas em computador Pentium III, 730 MHz e 128 MB RAM. Quanto as implementações utilizou-se o software MATLAB.

\subsection{Sistema de distribuição de 14 Barras}

O primeiro sistema teste (Cinvalar et al, 1988) possui uma subestação, 13 barras de carga, 13 trechos de rede, 3 alimentadores e tensão de 23 kV, veja Figura 4. Para esta análise inicial é considerada a possibilidade de instalação de apenas um único banco de capacitores de 150 kVAr em cada barra candidata do sistema de distribuição e e três níveis de carregamento: (i) carga leve ( 0,5 p.u); (ii) carga média (1,0 p.u); (iii) carga pesada $(2,45$ p.u).

Em relação ao carregamento do sistema, a metodologia não restringe o numero de patamares de carga. Entretanto, não sendo este ponto (níveis de carregamento) a contribuição principal do presente trabalho, adotou-se como solução global à solução encontrada para o nível médio de carregamento visto que este é o nível predominante (horas) ao longo da curva de carga.

Os limites mínimos e máximos de tensão adotados foram de 0,95 p.u e 1,05 p.u, respectivamente. Para a simulação referente ao nível pesado de carregamento permitiu-se um limite inferior de tensão de 0,9 p.u.

A tabela 1 é referente à solução do FPO para o caso base, onde são apresentados o período de carregamento, o valor

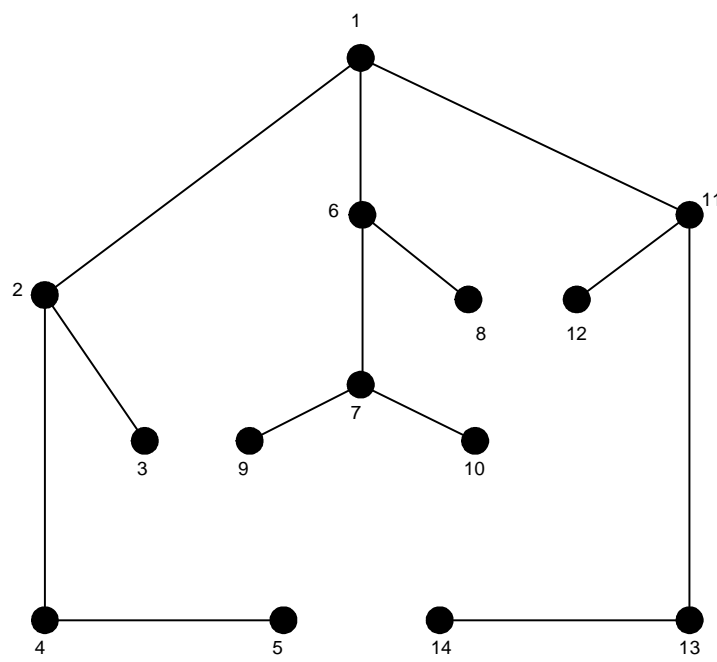

Figura 4: Sistema de Distribuição - 14 Barras.

Tabela 1: Solução Caso Base - Sistema 14 Barras.

\begin{tabular}{|c|c|c|c|}
\hline Carregamento & Leve & Médio & Pesado \\
\hline Período(horas) & 1000 & 6760 & 1000 \\
\hline Perdas(kW) & 255,7 & $1.083,6$ & $8.604,8$ \\
\hline Custo Total (US\$) & $\mathbf{1 5 . 3 4 2 , 0 0}$ & $\mathbf{4 3 9 . 5 0 8 , 0 0}$ & $\mathbf{5 1 6 . 2 8 8 , 0 0}$ \\
\hline
\end{tabular}

das perdas totais nos alimentadores e os custos destas perdas para os níveis de carregamento adotados.

A tabela 2 apresenta as barras candidatas para a alocação dos bancos de capacitores e os valores das sensibilidades $S$ calculados através da equação (5).

Com as barras candidatas definidas inicia-se o processo de alocação dos bancos de capacitores para cada um dos níveis de carregamento. A tabela 3 apresenta os resultados obtidos através da aplicação do Algoritmo Heurístico Construtivo Proposto (AHCP). A última linha da tabela mostra o custo total para cada período de carga. Pode-se verificar na tabela 3 que apesar do maior investimento em banco de capacitores ser obtido na simulação do nível médio de carregamento, o mesmo apresenta a maior redução entre os custos totais quando comparado com os resultados apresentados na tabela 1. Isto se deve principalmente a longa duração deste período (6760 horas).

A fim de comparar os resultados obtidos pela metodologia proposta fez-se uso do algoritmo genético com os parâmetros genéticos citados anteriormente. É importante ressaltar que as simulações do AG ocorrem dentro do mesmo espaço de busca.

Para esta primeira análise, o algoritmo genético encontrou a 
Tabela 2: Espaço de Busca Proposto - Sistema 14 Barras.

\begin{tabular}{|c|c|c|c|}
\hline Barras & 6 & 7 & 8 \\
\hline$S$ & 0.0071 & 0.0045 & 0.0030 \\
\hline
\end{tabular}

Tabela 3: Resultado do AHCP - Sistema 14 Barras.

\begin{tabular}{|c|c|c|c|}
\hline Carregamento & Leve & Médio & Pesado \\
\hline Barras Selecionadas & - & 6,7 e 8 & 7 e 8 \\
\hline $\begin{array}{c}\text { Total Instalado } \\
(\mathrm{kVAr})\end{array}$ & - & 450 & 300 \\
\hline Perdas (kW) & 255,7 & $1.074,0$ & $8.565,7$ \\
\hline Custo Perdas (US\$) & $15.342,00$ & $435.614,40$ & $513.942,0$ \\
\hline Custo Bancos (US\$) & - & $1.800,00$ & $1.200,00$ \\
\hline Custo Total (US\$) & $\mathbf{1 5 . 3 4 2 , 0}$ & $\mathbf{4 3 7 . 4 1 4 , 4 0}$ & $\mathbf{5 1 5 . 1 4 2 , 0 0}$ \\
\hline
\end{tabular}

mesma solução apresentada pela metodologia proposta. Entretanto, devido às características intrínsecas de cada algoritmo, o AHCP necessita de apenas uma fração do tempo computacional gasto pelo AG, veja tabela 4. Isto ocorre porque na metodologia proposta o processo de alocação é feito passo a passo evitando desta forma a "explosão" combinatória inerente ao problema.

Tabela 4: AHCP x AG - Sistema 14 Barras.

\begin{tabular}{|c|c|}
\hline Algoritmo & Tempo \\
\hline Genético & $1,5 \mathrm{~min}$ \\
\hline Proposto & $10 \mathrm{seg}$ \\
\hline
\end{tabular}

Uma simulação adicional foi realizada considerando-se todas as barras do sistema como candidatas. Nesta simulação, tanto o AHCP quanto o AG encontraram a mesma solução, porém como já mencionado, o AHCP é computacionalmente mais rápido.

\subsection{Sistema de distribuição de 70 Barras}

Este sistema possui uma barra de subestação, 69 barras, 74 trechos de rede e tensão de $12,66 \mathrm{kV}$, o sistema é apresentado na figura 5 (Amasifen, 2003). Para este sistema é considerada a possibilidade da instalação de até três bancos de capacitores de $200 \mathrm{kVAr}$ em cada barra candidata do sistema de distribuição. Para esta análise será considerado apenas o nível médio de carregamento (100\%), uma vez que este nível foi considerado o mais relevante devido à duração de seu período (6760 horas). A solução do FPO para o caso base corresponde a $71,5 \mathrm{~kW}$ de perdas totais nos alimentadores, sendo o custo referente a estas perdas de US\$29.000,40.
A análise deste sistema tem como objetivo principal verificar a qualidade da solução e o esforço computacional do algoritmo proposto. Isto porque o número total de combinações para este sistema é da ordem de $4^{70} \cong 10^{42}$, mostrando a dificuldade da obtenção de soluções ótimas e o elevado tempo computacional.

Como candidatas à alocação dos capacitores foram selecionadas através do índice dado pela equação (5), o conjunto de 17 barras apresentado na tabela 5 .

Tabela 5: Espaço de Busca Proposto - Sistema 70 Barras.

\begin{tabular}{|c|}
\hline Barras Candidatas a Alocação \\
\hline $10-11-12$-13-16-17-18-21-22-48-49-61-62-64-65-68-69 \\
\hline
\end{tabular}

Com as barras candidatas definidas inicia-se o processo de alocação dos bancos de capacitores. A tabela 6 apresenta o tempo computacional e os outros resultados obtidos pelo algoritmo heurístico construtivo proposto. Para este caso, os bancos de $200 \mathrm{kVAr}$ foram alocados da seguinte maneira: 1 banco na barra 12, 17, 18, 21 e 64; e 2 bancos na barra 61, totalizando 1400 kVAr inseridos no sistema de distribuição de energia.

Através da figura 6 é possível verificar a redução passo a passo das perdas ativas totais nos alimentadores com as in-

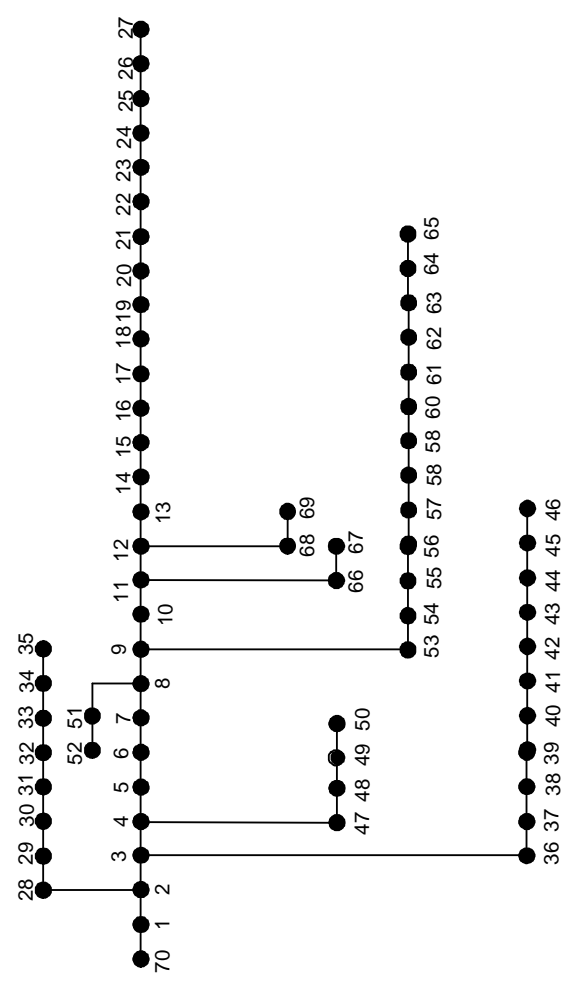

Figura 5: Sistema de Distribuição - 70 Barras. 
Tabela 6: Resultado do AHCP - Sistema 70 Barras

\begin{tabular}{|c|c|}
\hline Carregamento & Médio \\
\hline Barras Selecionadas & $12,17,18,21,61$ e 64 \\
\hline Total Instalado(kVAr) & 1400 \\
\hline Perdas (kW) & 16,77 \\
\hline Custo Perdas (US\$) & $6.801,92$ \\
\hline Custo Bancos (US\$) & $5.600,00$ \\
\hline Custo Total (US\$) & $12.401,92$ \\
\hline Tempo & 5 min \\
\hline
\end{tabular}

clusões de cada um dos bancos de 200 kVAr ao sistema de distribuição. Pode-se verificar através do comportamento do gráfico que a redução das perdas tende a diminuir com a inclusão de novos bancos até um determinado ponto (1400 $\mathrm{kVAr}$ ) onde qualquer acréscimo de potência reativa ao sistema faz com que as perdas ativas aumentem. Com a instalação total dos 1400 kVAr tem-se uma redução de aproximadamente $76,5 \%$ das perdas em relação ao caso base. Adicionalmente, destaca-se que foram necessárias apenas 15 simulações do problema de otimização (3) para a obtenção do resultado.

A fim de verificar a solução obtida pela metodologia proposta foram realizadas várias simulações do algoritmo genético considerando-se variações do número de indivíduos e usando o espaço de busca proposto.

A tabela 7 apresenta o custo total (CT) e o tempo computacional gastos pelo AG para variações do número de indivíduos, mostrando que quanto maior o número de indivíduos, maior a probabilidade de se encontrar soluções de melhor qualidade.

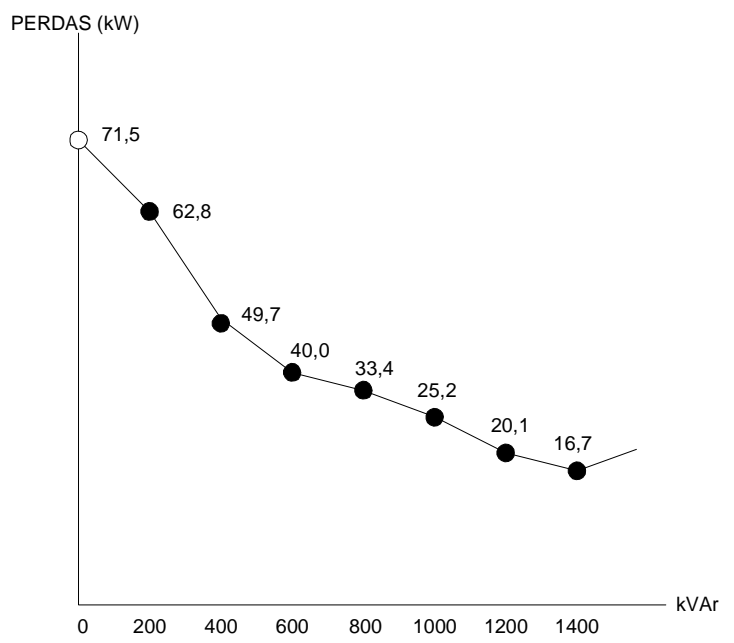

Figura 6: Gráfico Evolutivo das Perdas - 70 Barras.
Entretanto, com aumento significativo do tempo computacional. Pode-se notar que a partir de um determinado número de indivíduos (150), o AG apresenta a mesma solução encontrada pelo AHCP e para um número menor de indivíduos, o AG apresenta soluções de pior qualidade, mínimos locais.

Tabela 7: Simulações do AG - Sistema 70 barras

\begin{tabular}{|c|c|c|c|}
\hline $\begin{array}{c}\mathrm{N}^{o} \text { de } \\
\text { Indivíduos }\end{array}$ & $\begin{array}{c}\mathrm{N}^{o} \text { de } \\
\text { Simulações }\end{array}$ & Tempo & CT (US\$) \\
\hline 50 & 5.000 & $10 \mathrm{~min}$ & $12.895,36$ \\
\hline 100 & 10.000 & $23 \mathrm{~min}$ & $12.672,38$ \\
\hline 150 & 15.000 & $38 \mathrm{~min}$ & $12.401,92$ \\
\hline 200 & 20.000 & $51 \mathrm{~min}$ & $12.401,92$ \\
\hline 300 & 30.000 & $95 \mathrm{~min}$ & $12.401,92$ \\
\hline
\end{tabular}

Outras simulações foram realizadas utilizando-se todas as barras do sistema como candidatas à alocação dos bancos de capacitores, ou seja, sem redução do espaço de busca. A tabela 8 resume os resultados encontrados pelo AHCP e AG para o sistema 70 barras sem redução do espaço de busca. Os parâmetros genéticos utilizados, assim como as características do AG para está análise são os mesmos apresentados no item 6- Estudo de Casos.

Tabela 8: AHCP x AG - Sistema 70 Barras

\begin{tabular}{|c|c|c|}
\hline Algoritmo & Tempo & Custo Total \\
\hline Genético & $60 \mathrm{~min}$ & US\$ $17.238,41$ \\
\hline AHCP & $13 \mathrm{~min}$ & US\$ $15.355,92$ \\
\hline
\end{tabular}

Através dos resultados pode-se observar que em ambas metodologias o tempo computacional aumentou e a qualidade da solução diminuiu. Isto se deve principalmente ao aumento do número de soluções possíveis de investimento. Este fato mostra a importância do uso do índice proposto para redução do espaço de busca.

A garantia de sucesso, obtenção do ponto de mínimo global, só pode ser conseguida se todas as enumerações possíveis forem obtidas, caso contrário o que existem são probabilidades que dependem fatores intrínsecos a cada metodologia. Desta forma, o fato do AHC proposto ter apresentado uma solução mais econômica do que a obtida pelo AG é factível, embora se saiba que a probabilidade de sucesso das metaheuristicas seja maior devido à forma com que estes métodos percorrem o espaço de busca.

Como pode ser observado o esforço computacional da metodologia proposta é baixo quando comparado com o AG. Portanto, o AHCP pode ser utilizado várias vezes pelo ope- 
rador/planejador do sistema a fim de encontrar diversas configurações que possam ser aplicadas na prática. Em sistemas reais, devido a grande dimensão, as vantagens da metodologia proposta tornam-se ainda mais evidentes.

\section{CONCLUSÃO}

Este artigo apresentou uma proposta de algoritmo heurístico construtivo para o problema referente à alocação ótima de capacitores em sistemas de distribuição. Através dos resultados obtidos, os seguintes aspectos podem ser enfatizados:

- Os resultados iniciais obtidos podem ser considerados promissores, uma vez que o algoritmo proposto foi capaz de conciliar baixo esforço computacional com soluções de qualidade;

- A redução do espaço de busca usando multiplicadores de Lagrange associado à potência reativa local mostrouse um critério eficiente;

- A utilização da função sigmoidal para a modelagem do chaveamento (0-1) dos bancos associada à heurística adotada resultou em um critério eficiente de decisão.

- O algoritmo heurístico construtivo proposto também pode ser utilizado como gerador de soluções iniciais para a utilização de metas-heurísticas, como o AG, pois a eficiência do processo de busca do AG é função das soluções iniciais factíveis.

- Devido às características da metodologia adotada, o algoritmo proposto pode ser uma ferramenta bastante útil ao problema de alocação de bancos de capacitores. $\mathrm{O}$ algoritmo apresenta baixo esforço computacional, podendo ser utilizado várias vezes pelo operador do sistema a fim de encontrar configurações que possam ser aplicadas em sistemas reais.

- A utilização de outros valores para os parâmetros genéticos, outras formas de cruzamento e seleção dos indivíduos e a inicialização da população de forma não aleatória podem aumentar a eficiência do AG na obtenção de soluções de melhor qualidade.

- É importante ressaltar que nenhuma metodologia garante o ponto de mínimo global, ponto este que só é garantido através da enumeração completa de todas as alternativas de investimento.

\section{AGRADECIMENTOS}

Os autores gostariam de agradecer ao CNPq pelo apoio a esta pesquisa.

\section{REFERÊNCIAS}

Amasifen J.C.C.,"'Algoritmo Evolutivo Dedicado à Solução do Problema de Reconfiguração de Sistemas de Distribuição Radiais", Dissertação de Mestrado, UNESP 2003.

Baran, M. E. and Wu, F. F. (1989). Optimal Capacitor Placement on Radial Distribution Systems. IEEE Trans. on Power Delivery (January), Vol. 4, pp. 725-734.

Civanlar,S.; Grainger J.J.; Yin H. and Lee S.S.H (1988) .Distribution Reconfiguration for Loss Reduction. IEEE Transactions on Power Delivery, Vol. 3, No. 3, pp. 1217-1223.

Delfanti, M., Granelli, G.P., Marannino, P., Montagna, M. (2000). Optimal capacitor placement using deterministic and genetic algorithms. IEEE Transactions on Power Systems (August), Vol.15, n³, pp. 1041-1046.

Gallego, R. A., Monticelli, A.J., Romero, R. (2001). Optimal Capacitor Placement in Radial Distribution Networks. IEEE Transactions on Power Systems (November), Vol.16, n ${ }^{\circ}$, pp 235-243.

Garver L. L. (1970) .Transmission Network Estimation Using Linear Programming. IEEE Trans. on Power Systems (September), Vol. 89, $\mathrm{n}^{o}$ 7, pp.1688-1697.

Gomes, Flávio Vanderson., Carneiro Junior, Sandoval., Pereira, J. L. R., Vinagre, Márcio De Pinho., Garcia, Paulo Augusto Nepomuceno., Oliveira, Edimar José De., Araújo, Leandro Ramos (2005). A New Distribution System Reconfiguration Approach Using Optimal Power Flow Technique and Sensitivity Analysis for Loss Reduction. In: IEEE - PES - General Meeting, San Francisco. IEEE - Power Engineering Society (August), Vol. 1, $\mathrm{n}^{o} 1$, pp. 1-5.

Grainger, J.J and Lee, S.H (1981). Optimum Size and Location of Shunt Capacitors for Reduction of Losses on Distribution Feeders. IEEE Trans. on Power Apparatus and Systems (March), Vol. PAS-100, $\mathrm{n}^{\circ} 3$, pp.11051116.

Grainger, J.J and Lee, S.H (1982) .Capacity Release by Shunt Capacitor Placement on Distribution Feeders: A new Voltage Dependent. IEEE Trans. on Power Apparatus and Systems (May), Vol. PAS-101, no 5, pp. 1236-1244.

Granville, S., Melo, J.C.O., Melo, A.C.G (1996) .Application of Interior Point Methods to Power Flow Unsolvability. IEEE Transactions on Power Systems (May), Vol.11, $\mathrm{n}^{o} .2, \mathrm{pp} .1096-1103$.

Haykin, S. (1991). Neural Networks: A Comprehensive Foundation, Prentice Hall; 2nd edition. 
Huang, Y.C., Yang, H.T., Huang, C.L (1996). Solving the Capacitor Placement Problem in a Radial Distribution System Using Tabu Search Approach. IEEE Transactions on Power Systems (November), Vol.11, $\mathrm{n}^{\circ} 4$, pp. 1868-1873.

Karmakar, N.K. (1991). Computational Results of an Interior Point Algorithm for Large Scale Linear Programming. Mathematical Programming, 52, pp. 555-586.

Lee, S.H and Grainger, J.J (1981) .Optimum Placement of Fixed and Switched Capacitors on Primary Distribution. IEEE Trans. on Power Apparatus and Systems (January), Vol. PAS-100, ${ }^{o} 1$, pp. 345-352.

Levitin, G., Kalyuzhny, A., Shenkman, A., Chertkov, M (2000) .Optimal capacitor allocation in distribution systems using a genetic algorithm and a fast energy loss computation technique. IEEE Transactions on Power Delivery (April), Vol. 15, $\mathrm{n}^{\circ}$ 2, pp.623-628.

Monticelli A., Junior, A. S., Pereira, M. V. F., Cunha, S. H., Parker, B. J., Praca, J. C. G (1982) .Interactive Transmission Network Planning Using a Least-Effort Criterion. IEEE Trans. on Power Systems (October), Vol. 101, n ${ }^{o} 10$, pp. 3919-3925.

Oliveira, Edimar José De., Silva Junior, Ivo Chaves., Pereira, J. L. R., Carneiro Junior, Sandoval (2005). Transmission System Expansion Planning Using a Sigmoid Function to Handle Integer Investment Variables. IEEE Transactions on Power Systems (August), Vol. 1, $\mathrm{n}^{\circ} 1$, pp. 1-7.

Salama,H.N and Chikhani, M.M (2000).Classification of Capacitor Allocation Techniques. IEEE Transactions on Power Delivery (January), Vol.15, n ${ }^{\circ}$ 1, pp.387-392.

Santos, J.R., Exposito, A.G., Ramos, J.L.M (2004).

A reduced-size genetic algorithm for optimal capacitor placement on distribution feeders. Electrotechnical Conference, 2004 (May). Proceedings of the 12th IEEE Mediterranean, Vol.3, pp.963-966.

Villasana R., Garver L. L., Salon, S. J (1985) .Transmission Network Planning Using Linear Planning. IEEE Trans. on Power Systems (February), Vol. 104, n ${ }^{\circ}$ 2, pp.349356.

\section{APÊNDICE A - SISTEMA DE 70 BARRAS}

\begin{tabular}{|c|c|c|c|}
\hline De & Para & $\overline{\mathbf{R}(\Omega)}$ & $\mathbf{X}(\Omega)$ \\
\hline 70 & 1 & 0.0005 & 0.0012 \\
\hline 1 & 2 & 0.0005 & 0.0012 \\
\hline 2 & 3 & 0.0001 & 0.0001 \\
\hline 3 & 4 & 0.0015 & 0.0036 \\
\hline 4 & 5 & 0.0251 & 0.0294 \\
\hline 5 & 6 & 0.3660 & 0.1864 \\
\hline 6 & 7 & 0.3811 & 0.1941 \\
\hline 7 & 8 & 0.0922 & 0.0470 \\
\hline 8 & 9 & 0.0493 & 0.0251 \\
\hline 9 & 10 & 0.8190 & 0.2707 \\
\hline 10 & 11 & 0.1862 & 0.0619 \\
\hline 11 & 12 & 0.7114 & 0.2351 \\
\hline 12 & 13 & 1.0300 & 0.3400 \\
\hline 13 & 14 & 1.0440 & 0.3450 \\
\hline 14 & 15 & 1.0580 & 0.3496 \\
\hline 15 & 16 & 0.1966 & 0.0650 \\
\hline 16 & 17 & 0.3744 & 0.1238 \\
\hline 17 & 18 & 0.0047 & 0.0016 \\
\hline 18 & 19 & 0.3276 & 0.1083 \\
\hline 19 & 20 & 0.2106 & 0.0696 \\
\hline 20 & 21 & 0.3416 & 0.1129 \\
\hline 21 & 22 & 0.0140 & 0.0046 \\
\hline 22 & 23 & 0.1591 & 0.0526 \\
\hline 23 & 24 & 0.3463 & 0.1145 \\
\hline 24 & 25 & 0.7488 & 0.2475 \\
\hline 25 & 26 & 0.3089 & 0.1021 \\
\hline 26 & 27 & 0.1732 & 0.0572 \\
\hline 2 & 28 & 0.0044 & 0.0108 \\
\hline 28 & 29 & 0.0640 & 0.1565 \\
\hline 29 & 30 & 0.3978 & 0.1315 \\
\hline 30 & 31 & 0.0702 & 0.0232 \\
\hline 31 & 32 & 0.3510 & 0.1160 \\
\hline 32 & 33 & 0.8390 & 0.2816 \\
\hline 33 & 34 & 1.7080 & 0.5646 \\
\hline 34 & 35 & 1.4740 & 0.4873 \\
\hline 3 & 36 & 0.0044 & 0.0180 \\
\hline 36 & 37 & 0.0640 & 0.1565 \\
\hline 37 & 38 & 0.1053 & 0.1230 \\
\hline 38 & 39 & 0.0304 & 0.0355 \\
\hline 39 & 40 & 0.0018 & 0.0021 \\
\hline
\end{tabular}




\begin{tabular}{|c|c|c|c|}
\hline De & Para & $\mathbf{R}(\Omega)$ & $\mathbf{X}(\Omega)$ \\
\hline 40 & 41 & 0.7283 & 0.8509 \\
\hline 41 & 42 & 0.3100 & 0.3623 \\
\hline 42 & 43 & 0.0410 & 0.0478 \\
\hline 43 & 44 & 0.0092 & 0.0116 \\
\hline 44 & 45 & 0.1089 & 0.1373 \\
\hline 45 & 46 & 0.0009 & 0.0012 \\
\hline 4 & 47 & 0.0034 & 0.0084 \\
\hline 47 & 48 & 0.0851 & 0.2083 \\
\hline 48 & 49 & 0.2898 & 0.7091 \\
\hline 49 & 50 & 0.0822 & 0.2011 \\
\hline 8 & 51 & 0.0928 & 0.0473 \\
\hline 51 & 52 & 0.3319 & 0.1114 \\
\hline 9 & 53 & 0.1740 & 0.0886 \\
\hline 53 & 54 & 0.2030 & 0.1034 \\
\hline 54 & 55 & 0.2842 & 0.1447 \\
\hline 55 & 56 & 0.2813 & 0.1433 \\
\hline 56 & 57 & 1.5900 & 0.5337 \\
\hline 57 & 58 & 0.7837 & 0.2630 \\
\hline 58 & 59 & 0.3042 & 0.1006 \\
\hline 59 & 60 & 0.3861 & 0.1172 \\
\hline 60 & 61 & 0.5075 & 0.2585 \\
\hline 61 & 62 & 0.9740 & 0.0496 \\
\hline 62 & 63 & 0.1450 & 0.0738 \\
\hline 63 & 64 & 0.7105 & 0.3619 \\
\hline 64 & 65 & 1.0410 & 0.5302 \\
\hline 11 & 66 & 0.2012 & 0.0611 \\
\hline 66 & 67 & 0.0047 & 0.0014 \\
\hline 12 & 68 & 0.7394 & 0.2444 \\
\hline 68 & 69 & 0.0047 & 0.0016 \\
\hline 11 & 43 & 0.5000 & 0.5000 \\
\hline 13 & 21 & 0.5000 & 0.5000 \\
\hline 15 & 46 & 1.0000 & 1.0000 \\
\hline 50 & 59 & 2.0000 & 2.0000 \\
\hline 27 & 65 & 1.0000 & 1.0000 \\
\hline & & & \\
\hline
\end{tabular}

Tabela-A.2: Dados das Barras de Carga

\begin{tabular}{|c|c|c|c|c|c|}
\hline Barra & $(\mathbf{k W})$ & $\mathbf{k V A r})$ & Barra & $\mathbf{( k W})$ & $\mathbf{( k V A r})$ \\
\hline 6 & 0.8780 & 0.7200 & 37 & 8.6670 & 6.1850 \\
\hline 7 & 13.4550 & 0.7200 & 39 & 8.0000 & 5.7090 \\
\hline 8 & 24.8770 & 17.810 & 40 & 8.0000 & 5.7090 \\
\hline 9 & 10.0000 & 7.2080 & 41 & 0.3920 & 0.3250 \\
\hline 10 & 9.3330 & 6.6660 & 43 & 2.0000 & 1.4270 \\
\hline 11 & 48.5000 & 34.6090 & 45 & 3.0760 & 8.7870 \\
\hline 12 & 48.5000 & 34.6090 & 46 & 3.0760 & 8.7870 \\
\hline 13 & 2.7100 & 1.8210 & 48 & 26.3500 & 18.8000 \\
\hline 14 & 2.7100 & 1.5210 & 49 & 28.2260 & 91.4920 \\
\hline 16 & 15.1760 & 10.1980 & 50 & 128.2260 & 91.4920 \\
\hline 17 & 16.5000 & 11.7750 & 51 & 13.5120 & 0.8940 \\
\hline 18 & 16.5000 & 11.7750 & 52 & 1.2020 & 1.1620 \\
\hline 20 & 0.3160 & 0.2120 & 53 & 1.4490 & 1.1620 \\
\hline 21 & 37.9830 & 27.1000 & 54 & 8.7870 & $6 ; 3220$ \\
\hline 22 & 1.7620 & 1.1840 & 55 & 8.0000 & 5.7080 \\
\hline 24 & 9.3900 & 6.6700 & 59 & 0.6670 & 24.0250 \\
\hline 26 & 4.6670 & 3.3300 & 61 & 414.6670 & 295.910 \\
\hline 27 & 4.6670 & 3.3300 & 62 & 10.6670 & 7.6120 \\
\hline 28 & 8.6670 & 6.1850 & 64 & 75.6700 & 53.8730 \\
\hline 29 & 8.6670 & 6.1850 & 65 & 19.6700 & 13.9120 \\
\hline 33 & 4.5820 & 3.2600 & 66 & 6.0000 & 4.2820 \\
\hline 34 & 6.5010 & 5.5490 & 67 & 6.0000 & 4.2820 \\
\hline 35 & 1.9200 & 1.2900 & 68 & 9.3000 & 26.700 \\
\hline 36 & 8.6670 & 6.1850 & 69 & 9.3000 & 26.700 \\
\hline
\end{tabular}

